\title{
Les lits en ivoire et les lits en os du Musée archéologique de Naples
}

The ivory and bone beds of the Archaeological Museum of Naples

\section{Anselme Cormier}

\section{(2) OpenEdition}

\section{Journals}

Édition électronique

URL : http://journals.openedition.org/cefr/4367

DOI : $10.4000 /$ cefr.4367

ISSN : 2282-5703

Éditeur

École française de Rome

Référence électronique

Anselme Cormier, "Les lits en ivoire et les lits en os du Musée archéologique de Naples », Chronique des activités archéologiques de l'École française de Rome [En ligne], Les cités vésuviennes, mis en ligne le 20 mai 2020, consulté le 20 mai 2020. URL : http://journals.openedition.org/cefr/4367 ; DOI :

https://doi.org/10.4000/cefr.4367

Ce document a été généré automatiquement le 20 mai 2020.

(c) École française de Rome 


\title{
Les lits en ivoire et les lits en os du Musée archéologique de Naples
}

The ivory and bone beds of the Archaeological Museum of Naples

\author{
Anselme Cormier
}

\section{NOTE DE L'AUTEUR}

Cette présentation des éléments en ivoire et en os de lits provenant de Pompéi illustre un des aspects du module de recherche intitulé « Ad luxuriae instrumenta. Les objets dans leur contexte, entre la demeure et la tombe : le mobilier en ivoire et en os ouvragé à Pompéi ", centré sur l'étude de l'art et de l'artisanat de l'ivoire et de l'os à l'échelle de la ville. Ce programme s'insère dans l'axe 2 « Espaces, économie et artisanat dans les cités vésuviennes (l'artisanat urbain et la vie économique antique à Pompéi) » du Centre Jean Bérard, et je remercie à ce titre Mesdames Claude Pouzadoux et Priscilla Munzi. La recherche a été financée grâce aux crédits du Ministère de l'Europe et des Affaires étrangères dans le cadre de la Mission archéologique « Italie du Sud » (Paris). Il constitue également une part du projet déjà initié de création d'un catalogue raisonné exhaustif des ivoires et des os du Musée archéologique de Naples.

Je remercie le Directeur du Musée, Monsieur Paolo Giulierini, Madame Marialucia Giacco, funzionario archeologo conservatore e Responsabile Ufficio Mostre Italia/Ester au sein de la direction scientifique, ainsi que Madame Floriana Miele, funzionario archeologo conservatore e Responsabile Ufficio Catalogo, et Monsieur Andrea Milanese, Directeur de l'Ufficio di Museologia e Documentazione storica, pour leur disponibilité et leur aide. Je remercie également Madame Maria Morisco, les consegnatari du musée et le photographe Giorgio Albano, qui se révèlent à tout moment de formidables facilitateurs. 


\section{Introduction}

1 L'histoire de la redécouverte de Pompéi au XVIII siècle, puis les épisodes successifs qui ont construit - ou reconstruit - son histoire, intéressent les chercheurs au point d'être devenus des sujets d'étude propres ${ }^{1}$, indissociables maintenant des nouvelles fouilles archéologiques et recherches, qu'ils éclairent d'une manière très utile. Il en est ainsi de l'ivoire et de l'os ouvragé : au fil des rayonnages sans fin des réserves du Musée archéologique de Naples, une cellule conserve des cassettes dans lesquelles sont entreposés les artefacts en ivoire et en os mis au jour entre le XVII et le début du XX siècle, et provenant de divers sites de Campanie comme Herculanum, Cumes, Pouzzoles, Quarto, Capoue, Lacedonia ou encore Pietrabbondante (Molise) - du moins pour ce qui concerne les lieux pour le moment identifiés et répertoriés, sans compter tous les objets et fragments dont l'origine est soit non assurée soit perdue. Pour l'immense majorité cependant, ils proviennent de Pompéi.

2 L'étude de lits funéraires ornés d'ivoire découverts dans d'importants mausolées d'une des nécropoles de $\mathrm{Cumes}^{2}$, ajoutée à celle d'autres contextes archéologiques du même lieu$^{3}$, ainsi qu'à l'observation du mobilier provenant d'autres cités, a mis en évidence l'importance que revêtaient l'ivoire et l'os non seulement dans le cérémonial et le mobilier funéraires, mais aussi par voie de conséquence dans la vie quotidienne des vivants, puisque les objets retrouvés dans les tombes en proviennent. Tant cette articulation entre funéraire et domestique que le reflet du second dans le premier, évoqué par les chercheurs ${ }^{4}$, ont fait naître en 2017 l'idée de proposer à l'échelle de Pompéi cette fois une clarification morphologique, typologique et iconologique, non seulement pour ce qui concerne les lits domestiques et funéraires, mais aussi pour les autres types d'artefacts en matière dure animale, afin de développer une étude d'ensemble sur le travail de l'os et de l'ivoire, et d'aborder cet aspect important de la culture matérielle à l'échelle d'une ville romaine, et qui concerne tous les niveaux de l'échelle sociale. À ce titre, Pompéi constitue un exemple particulièrement représentatif.

\section{Contexte de travail}

3 Depuis 2017, et désormais dans le cadre d'une collaboration initiée en 2019 entre le Centre Jean Bérard et le Musée archéologique de Naples, et celle en préparation entre le Centre Jean Bérard et l'université libre de Bruxelles, ce travail de recherche s'oriente autour de trois axes: d'une part, l'étude de l'art et de l'artisanat de l'ivoire et de l'os à Pompéi, sous les aspects archéologique, historique et sociologique ${ }^{5}$; celle, d'autre part, de l'ivoire comme matériau de luxe, porteur de symboliques particulières, et de l'os comme son pendant bon marché, d'approvisionnement facile en matière première et par conséquent beaucoup plus répandu ; la création, enfin, d'un catalogue raisonné et exhaustif des pièces du musée, à partir de la réalisation d'un inventaire passant par une systématisation du traitement des objets, des tris et des rapprochements, ainsi que par une étude des techniques du travail artisanal utilisées, ayant pour objectif la constitution de corpus cohérents, en vue de l'élaboration de typologies fonctionnelles.

Les publications relatives aux collections du musée sont de fait peu nombreuses: en 1963, A. De Franciscis publiait quelques éléments marquants en ivoire et en ${ }^{6}{ }^{6}$; en 1986, M.R. Borriello proposait un chapitre sur Gli avori et gli ossi lavorati dans un 
catalogue du musée ${ }^{7}$, dans lequel elle inventoriait et décrivait vingt-six pièces de valeur particulièrement significative, mais là aussi en nombre restreint ; en 2007, E. De Carolis publiait une classification typologique sur Letti, tavoli, sedie e armadi, confrontée aux sources littéraires et iconographiques ${ }^{8}$. Différents articles et monographies traitant d'autres sujets évoquent des pièces éparses, sans aborder la question d'ensemble.

5 Au Musée de Naples, le travail d'inventaire permet de dresser la liste des objets qui, avec le temps, auraient perdu leur identifiant et de ceux qui pourraient faire l'objet d'une restauration. Ce catalogue repose sur la consultation des trois inventaires historiques successifs, initiés chacun au XIX siècle $^{9}$ : l'Inventario Arditi, du Real Museo Borbonico (1826), l'Inventario Sangiorgio (1852) et l'Inventario Fiorelli, l'Inventario Generale Soprintendenza Antichità Campania (1863 à 1871). Bien que chaque inventaire soit intégré dans le suivant il est nécessaire, pour les objets découverts dans les premiers temps des dégagements, de tous les consulter, en particulier parce que les numéros d'inventaire ont pu parfois changer, et aussi pour y retrouver des informations qui n'auraient pas été reportées. La reconstitution des contextes de découvertes s'appuie, quant à elle, sur les quelques informations fournies par les Archives historiques et les journaux de fouilles.

6 En complément des archives, les réserves du site même de Pompéi et les fouilles récentes offrent la possibilité de conduire cette approche archéologique contextuelle. Car sur place l'étude réalisée sur place à partir de 2017 et menée parallèlement à celle du Musée de Naples a fourni des premiers résultats qui permettent de lancer des ponts entre les différentes sources documentaires. Il existe en outre plusieurs études sur l'ivoire et l'os ouvragés conservés à Pompéi qui peuvent être rattachés aux contextes d'où ils proviennent. On peut citer par exemple le volume de Penelope M. Allison sur le matériel en os travaillé qui provient de l'Insula dite "du Ménandre $»^{10}$. Dans le domaine funéraire, les fouilles de la nécropole de Porta Nocera ont permis une étude particulièrement développée ${ }^{11}$, aujourd'hui reprise sur d'autres contextes de la nécropole qui livrent des informations extrêmement précises sur la présence des artefacts en ivoire et en os dans les bûchers et dans les tombes ${ }^{12}$.

7 À l'issue de ces premières campagnes d'étude sur le terrain, la base de données comprend actuellement un peu plus de 510 artefacts conservés dans les dépôts du musée, fragmentaires ou non, qui ont été étudiés et décrits, identifiés et classés selon leur typologie fonctionnelle ${ }^{13}$. Cette étape représente pour l'heure un cinquième d'une

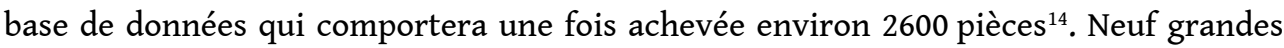
catégories composent pour le moment cet ensemble, et qui peuvent être subdivisée en plusieurs types :

- L'instrumentum et les outils : cuillères, manches, couteaux, stylets, fuseaux et fusaïoles, quenouilles, couteaux de tissage, aiguilles, poinçons, règles, instruments de mesure. - Les éléments d'ameublement : éléments de montants de lits (anneaux, bobines, cylindres figurés ou non, chapiteau, cloches), appliques de cadre de lit, appliques d'accotoirs de lit (couronnements de fulcrum, médaillons), éléments de charnière éléments de serrure, appliques décoratives.

- Les éléments de coffrets : placages et appliques décoratifs, pieds.

- Le petit mobilier : boîtes rectangulaires avec couvercle à glissière, pyxides, ayballes, unguentaria.

- Les éléments d'instruments de musique. 
- La parure : épingles, pendentifs, amulettes en pendentif, éléments d'éventail, éléments d'ombrelle, fibules.

- Les ustensiles de toilette : peignes, cure-oreilles, spatules/cuillères à parfum.

- Les éléments de jeu : jetons, dés, osselets.

- Les militaria : gardes de pugio, pommeaux.

\section{Les lits romains : formes et typologie}

8 Héritage de traditions anciennes déjà bien connues dans le monde méditerranéen, les lits à l'époque romaine ont connu un développement très varié de formes et de styles : ils constituent une sorte de point d'orgue et d'aboutissement par les variétés typologiques qu'ils ont développées à la fin de la République et sous le Haut-Empire ${ }^{15}$. On a coutume de situer chronologiquement ce que nous nommons lits de type romain ${ }^{16}$ entre la fin du $\mathrm{II}^{\mathrm{e}} \mathrm{s}$. av. et le milieu du II ${ }^{\mathrm{e}} \mathrm{s}$. apr. J.-C., si l'on se réfère aux occurrences connues par l'archéologie. Peu de modèles sont connus ensuite ${ }^{17}$, et ils n'apparaissent en tout cas plus dans les tombes, où ils sont progressivement remplacés par des représentations en relief sur les sarcophages - ce qui n'exclut naturellement pas leur présence logique dans les demeures, mais nous n'en connaissons pas d'exemplaire.

Parmi les différents matériaux utilisés pour la décoration, on trouvait bien sûr les bois plus ou moins précieux (peu en est conservé), mais aussi le bronze, l'or, l'argent, l'écaille de tortue, le stuc, la terre cuite, et bien sûr l'ivoire et l'os. Ces matériaux pouvaient être utilisés en placage recouvrant des supports en bois, ou bien directement comme armature décorée, comme ce peut être le cas pour le bronze ou même l'ivoire, que l'épaisseur de la matière première autorisait - contrairement à l'os, par nature moins épais. Rappelons très succinctement que l'os se travaillait selon des techniques appréhendées grâce essentiellement aux traces laissées par les outils sur les déchets de manufacture et sur le revers de certaines pièces finies - des stigmates sont visibles sur quelques éléments présentés $\mathrm{ici}^{18}{ }^{18}$, et grâce aux ateliers d'artisans retrouvés ${ }^{19}$. On procédait au débitage de la matière première par sciage, puis le façonnage s'effectuait au moyen d'un couteau ou d'un ciseau à tranchant lisse ou denté. L'intérieur des objets tubulaires était élargi au moyen d'une gouge, et le tournage des pièces arrondies se faisait sur un tour à rotation actionnée par un archet. On perforait grâce à un foret entraîné lui aussi par un archet. Enfin les finitions, motifs incisés et sculptures émergeaient de la matière sous l'action du couteau, du ciseau, de la râpe et de la lime.

La forme la mieux connue et la plus achevée du lit romain se composait de trois parties : les montants, le châssis et les accotoirs en forme de S, qui pouvaient être ornés de fulcra à leurs extrémités, sur les tranches. Les quatre montants étaient fixés aux angles du cadre ou, plus couramment, traversaient le châssis de part en part pour constituer sur le dessus, en une sorte de pommeau, les supports des accotoirs; leur solidité était généralement assurée par une âme de fer centrale sur laquelle étaient enfilés ou fixés les volumes et éléments décoratifs ${ }^{20}$. Les dimensions pouvaient varier, mais tout en restant manifestement dans des proportions comparables: elles sont estimées, en contexte funéraire, entre 180 et $200 \mathrm{~cm}$ de longueur, 60 et $80 \mathrm{~cm}$ de largeur et 40 à $70 \mathrm{~cm}$ de hauteur ${ }^{21}$. Profilés en forme de $\mathrm{S}$, les fulcra se composaient de trois éléments : le médaillon en partie inférieure, un champ en partie intermédiaire, et un couronnement en partie supérieure, prolongeant le champ central. Comme pour les deux autres parties du lit, les fulcra pouvaient être décorés de sculptures en haut ou 
bas-relief. Il existe cependant un certain nombre d'occurrences dont les montants ou le cadre n'ont conservé aucune décoration ${ }^{22}$.

11 C'est en fonction de ces trois parties et de leurs différents agencements morphologiques que peut être proposée une typologie précise ${ }^{23}$. Dans les lignes principales - pour lesquelles il existe des déclinaisons variées -, voici les formes que pouvaient prendre ces trois parties :

A. Les montants: A.1. éléments tubulaires tronconiques; A.2. éléments tubulaires cylindriques; A.3. éléments de disque; A.4. éléments constitutifs de volumes et de parties bombées (A.4.1. campaniformes, A.4.2. cupuliformes, A.4.3. sphériques plus ou moins aplatis, A.4.4. annulaires); A.5. éléments sculptés à décor figuré ; A.6. éléments sculptés ou incisés à décor géométrique ou végétal.

B. Le cadre: B.1. appliques et garnitures en bandeau; B.2. appliques circulaires décoratives; B.3. appliques décoratives figurées en relief; B.4. éléments sculptés ou incisés à décor géométrique ou végétal ; B.5. appliques lisses à contour géométrique.

c. Le fulcrum : C.1. partie basse : le médaillon ; C.2. partie centrale : le champ ; C.3. partie haute : le couronnement.

12 Matières dures animales de constitution et d'aspect qui les rapprochent à certains égards, l'ivoire et l'os sont la plupart du temps étudiés et traités ensemble, à juste titre souvent, par souci de commodité, et parce que ces deux matériaux servent à produire des types d'objets similaires. Afin cependant de signifier les différences visuelles qui peuvent les caractériser, ils sont présentés ici séparément, chacun par lots représentatifs. Pour ce qui est de l'ivoire, hormis la fabrication de petit mobilier qui n'utilise qu'une quantité limitée de matière première, souvent à partir de déchets, de morceaux de moindre qualité ou abîmés de défenses d'éléphant par exemple, il pouvait servir à la fabrication d'objets plus épais et de tailles bien plus conséquentes, et destinés à une clientèle nécessairement aisée, voire fortunée ${ }^{24}$.

\section{Les lits ornés d'os ouvragé (pl. 1)}


PI. 1 - Éléments en os de montant de lit : montant $\left(n^{\circ s} 1,2\right)$, cadre $\left(n^{\circ 5} 3,4,5\right)$, accotoir $\left(n^{\circ} 6\right.$ couronnement, $\mathrm{n}^{\circ} 7$ médaillon de fulcrum).

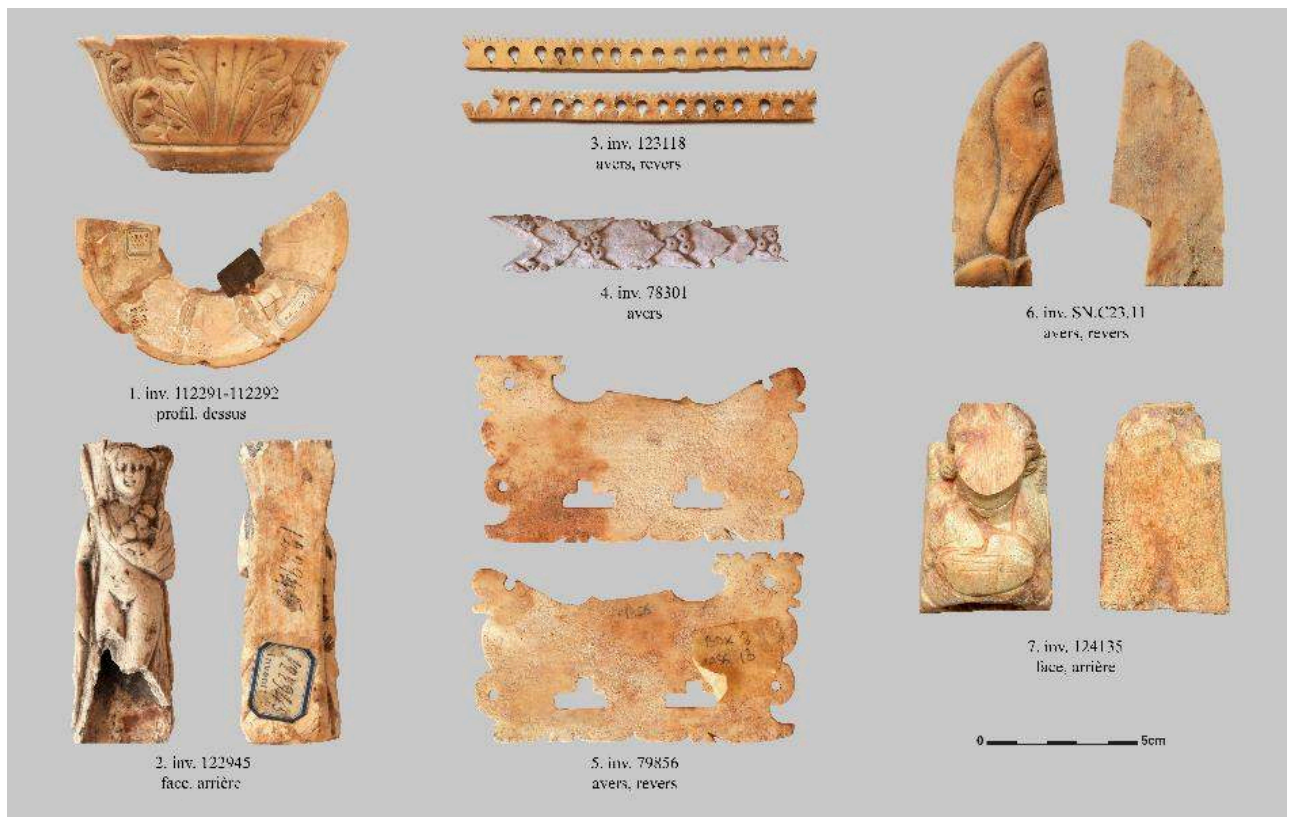

(c) A. Cormier, avec l'aimable autorisation du Musée archéologique de Naples.

D'un point de vue archéologique, le peu de données de contextualisation nous prive d'informations importantes, mais qui pourront être partiellement complétées par la consultation des journaux de fouilles. Toutefois, parmi les éléments ou groupes d'éléments en os pour le moment étudiées - comprenant tous les types de mobilier, comme précisé plus haut -, 43 appartiennent à des lits, dont certains sont présentés ici, selon leur type et leur emplacement sur la structure qu'ils décoraient ${ }^{25}$. Les sept pièces proposées ici proviennent de Pompéi. Des références comparatives sont indiquées pour chacune de ces pièces, publiées ou inédites.

\section{A. Les montants}

\section{A.4. Éléments constitutifs de volumes et de parties bombées}

\section{A.4.2. Élément cupuliforme}

14 Identification : chapiteau décoratif de montant de lit (pl. 1, $\mathrm{n}^{\circ} 1$ )

Inventaire général Fiorelli : 112291-112292

Provenance : Pompéi

Dimensions : L. max. 41 ; 1. max. X ; ép. max. 5,4 ; diam. int. max. 36 ; diam. ext. max. 9

Détermination : os

Description : cinq plaquettes complètes de forme trapézoïdale assemblées entre elles et constituant la moitié d'un volume en forme de cloche renversée. La partie inférieure est rehaussée d'une moulure de laquelle s'élancent vers le haut des ensembles végétaux avec tiges et feuilles, répétées côte à côte et ressemblant partiellement à des palmettes. Il s'agit probablement d'un chapiteau de montant de lit, qui était constitué d'un volume interne en bois sur lequel étaient fixées ces décorations.

Référence comparative : Cormier 2015, vol. II, p. 106 et 124. 


\section{A.5. Élément sculpté à décor figuré}

Identification : cylindre figuré de montant de lit (pl. 1, n $\left.{ }^{\circ} 2\right)$

Inventaire général Fiorelli : 122945

Provenance : Pompéi

Dimensions : H. max. 102 ; l. max 34 ; ép. max. 31

Détermination : os

Description : Pièce de diaphyse évidée et sculptée, s'élargissant vers la base, et dont la surface est composée de deux parties distinctes, à l'avant et à l'arrière. La partie avant, en bas-relief marqué, présente un personnage masculin en pied. La moitié des membres inférieurs a disparu, mais laisse voir des cuisses nues et charnues. Cette nudité se prolonge jusqu'au milieu du buste, en partie caché en diagonale par la tunique remontée sur l'épaule gauche puis jusqu'à l'épaule droite vers une attache (une fibule ?), en rejoignant par le dos l'autre côté. Le drapé plissé de la tunique, utilisé comme une besace en bandoulière, laisse dépasser plusieurs éléments de petite taille, assimilables à des fruits, et en particulier une grenade au premier plan. Il s'agit probablement de Priape, dont la représentation dans ce type de posture est attestée.

Références comparatives: Orsi 1912, fig. 17 p. 386 et Zambito 2010 p. 193 pour des similitudes dans la morphologie d'ensemble; voir surtout Rodziewicz 2007, pl. 86, n ${ }^{\circ} 4$ pour une possible identification équivalente.

\section{B. Le cadre}

\section{B.1. Appliques et garnitures en bandeau}

Identification : Applique décorative à décor végétal (pl. 1, $\left.\mathrm{n}^{\circ} 4\right)$

Inventaire général Fiorelli : 78301

Inventaire San Giorgio : 7881

Provenance : Pompéi

Dimensions : L. max. 101 ; l. $\max 19$; ép. max. 6

Détermination : os

Description : Élément longiforme dont la surface légèrement bombée est pourvue d'une décoration en emboîtement régulier de feuilles perlées. Le revers est lisse et plat. Il s'agit d'une partie constitutive de bandeau décoratif de cadre de lit, ou peut-être de coffret.

Références comparatives : Rossi - Savi 2012, fig. 11 p. 144 ; Caretta 2014, fig. 17 p. 135.

Identification : applique décorative ajourée en bandeau (pl. 1, n 3)

Inventaire général Fiorelli : 123118

Provenance : Pompéi

Année de découverte : 1862 ( 5 avril). Expédié au Musée de Naples le 17 juillet 1894.

Dimensions : L. max. 118 ; 1. max 10,3 ; ép. max. 2

Détermination : os

Description : Fin bandeau décoratif ajouré, percé d'une succession de trous en forme de goutte, en partie arrondie et en partie en coin, peut-être comblé par des éléments en pâte de verre. Un bord est rectiligne et l'autre dentelé.

Références comparatives: Aucune occurrence similaire n'a pour le moment été trouvée. 


\section{B.5. Applique lisse à contour géométrique}

18

Identification : applique décorative (pl. 1, $\left.\mathrm{n}^{\circ} 5\right)$

Inventaire général Fiorelli : 79856

Provenance : Pompéi

Dimensions : L. max. $111 ; 1$. max 63 ; ép. max. 4

Détermination : os

Description : Élément de placage décoratif plat et ajouré, dont les contours dessinent des motifs géométriques convexes et concaves symétriques. Deux des quatre angles sont brisés et incomplets. Quatre percements ronds sont disposés non loin des quatre angles, probablement destinés à accueillir des clous décoratifs de fixation. Le long d'un des côtés sont présents deux autres percements de forme ovoïdale étirée, ponctuée par un ressaut rectangulaire. Cet élément appartenait à un ensemble décoratif disposé sur le cadre d'un lit, ou bien peut-être sur la paroi d'un coffret.

Références comparatives : Faita 1989, pl. XIII ; Bovero 2014, p. 85.

\section{Le fulcrum}

\section{C.1. Partie basse : le médaillon}

Identification : applique décorative figurée de médaillon (pl. 1, nº 7)

Inventaire général Fiorelli : 124135

Provenance : Pompéi

Année de découverte : 1866 (6 octobre). Expédié au Musée de Naples le 19 mai 1896.

Dimensions : H. max. 67,4; 1. max 43,4 ; ép. max. 27,6

Détermination : os

Description : Élément sculpté en relief dans une diaphyse, constitutif d'un motif global était constitué à l'origine de plusieurs pièces. Un personnage en buste est représenté, presque complet : il ne manque que le relief du visage, qui a disparu. Le haut du tronc est couvert d'un vêtement dont les plis et ornements sont exprimés par des incisions linéaires, perpendiculaires et transversales. Les bras sont cachés par le vêtement. Au niveau de la tête, la chevelure ondule sur les parties latérales, remontant ensuite sur le dessus et derrière. La face arrière, plate, comporte des traces d'outillage, indiquant qu'il s'agit d'un placage destiné à une surface plane. Les bords latéraux sont également taillés en plats et lisses, perpendiculaires au revers. Le canal médullaire de l'os, intégralement présent, a permis de conserver une épaisseur. Il est à noter que cette pièce est constituée de plusieurs morceaux assemblés à l'origine, pour permettre d'augmenter l'épaisseur de la sculpture (la tête est en effet composée de deux éléments rajoutés à la diaphyse), mais aussi sa largeur. La disparition du visage rend l'identification du personnage difficile. Il s'agit en tout cas d'un placage décoratif de meuble, probablement de lit, et pouvait être situé soit sur le cadre soit plus probablement sur un médaillon de fulcrum.

Références comparatives : Colivicchi 2002, fig. 52, p. 318-319 ; Bovero 2014, p. 87.

\section{C.2. Partie haute : le couronnement}

Identification : Applique figurée de couronnement de fulcrum (pl. 1, nº 6)

Inventaire provisoire : SN.C23.11

Provenance : Pompéi

Chronique des activités archéologiques de l'École française de Rome , Les cités vésuviennes 
Dimensions : H. max. 81 ; 1 max 37 ; ép. max. 8,4

Détermination : os

Description : Élément incomplet figuré de placage de forme incurvée, pourvu d'une décoration en bas-relief représentant une tête d'oiseau aquatique, caractéristique d'un couronnement de fulcrum de lit. La partie supérieure montre une section transversale en biseau coupant la tête au niveau de l'œil en direction de la commissure du bec, qui indique la présence d'une autre pièce qui achevait l'ensemble. La partie inférieure comprend la double vague caractéristique (également incomplète) constitutive de la liaison avec le champ d'un fulcrum de lit.

Références comparatives : Copersino 2001, p. 246 ; Béraud - Gebara 1986, fig. 8 n²1-22. Voir également Bianchi 2010, qui répertorie un nombre important d'éléments de même type.

\section{Les lits ornés d'ivoire sculpté (pl. 2)}

PI. 2 - Éléments en ivoire de montant de lit : montant $\left(n^{\circ s} 1,2,3\right)$, cadre $\left(n^{\circ s} 4,5\right)$, accotoir $\left(n^{\circ}\right.$ 6 couronnement, $n^{\circ} 7$ médaillon de fulcrum).

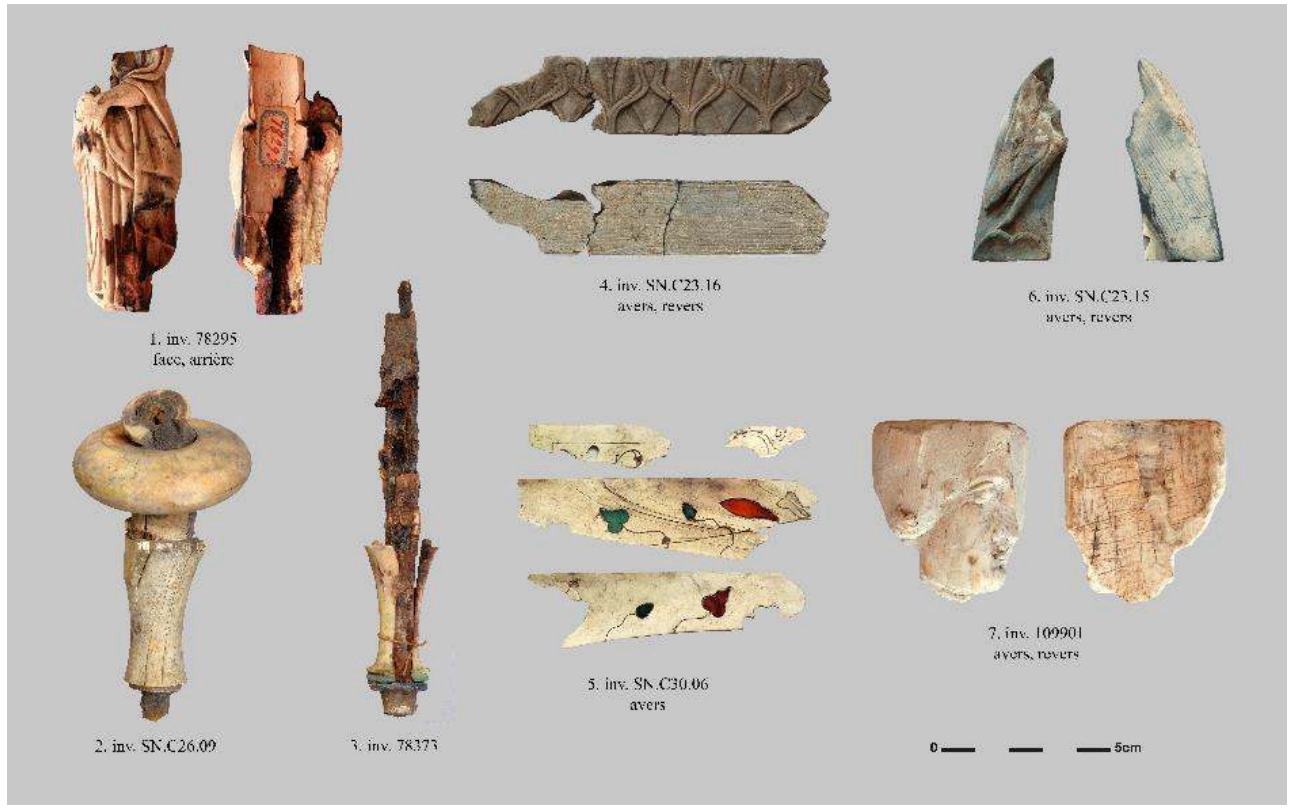

(c) A. Cormier, avec l'aimable autorisation du Musée archéologique de Naples.

Sans surprise, les éléments en ivoire sont beaucoup moins nombreux que ceux en os. Sur l'ensemble pour le moment étudié, 103 pièces ou groupes de fragments sont déterminés comme tels, dont un tiers environ appartiennent à des lits, même si un doute subsiste pour quelques-uns. Toutes les parties du lit sont représentées, provenant de contextes épars : montants pour la majorité, cadre, et fulcra en petit nombre. La cassette $n^{\circ} 26$ présente un intérêt tout particulier: elle concentre un ensemble de 83 pièces en ivoire appartenant à des montants de lits, encore enfilées sur des fragments des âmes de fer internes servant d'armature, qui comportent également des restes de bois. Leur état très détérioré ne permet pas encore de déterminer les liens qui existent entre elles, et de combien de lits différents elles proviennent, mais un 
nettoyage et un examen plus attentif permettra d'en dire davantage. Sept pièces sont présentées ici, réparties de la même manière que pour l'os.

\section{A. Les montants}

\section{A.1. Élément tubulaire tronconique}

Identification : partie basse de montant de lit (pl. 2, $\mathrm{n}^{\circ} 3$ )

Inventaire général Fiorelli : 78373

Inventaire San Giorgio : 10160

Provenance : Pompéi

Dimensions : H. max. 127,9 ; diam. ext. max. 19,6

Détermination : ivoire, bois, fer, alliage cuivreux

Description : élément fragmentaire longiforme de montant de meuble, composé d'une âme de fer en partie interne, qui repose sur le pied proprement dit en fer (cylindre et anneau de soutènement). Sur ce socle repose une moulure en double listel initiant un élément tubulaire tronconique fragmentaire, lui-même s'élargissant vers le haut pour se terminer en arête, avec ressaut interne en forme de tenon sur la tranche. L'élément qui s'emboîtait au-dessus n'a conservé que la base interne, en moignon encore enfilé sur l'âme de fer. Probablement s'agissait-il d'une bobine. L'âme de fer, d'environ $5 \mathrm{~mm}$ de diamètre mais fortement corrodée, démarre avec le cylindre et l'anneau du pied, puis se prolonge encore vers le haut, rehaussé de restes ligneux, avant d'atteindre la brisure.

Références comparatives : de nombreux fragments comparables sont en cours d'étude au Musée archéologique de Naples et dans les réserves du site de Pompéi, dont surtout inv. 78343, mais aussi 78358, 78372, 78375, 78387, ainsi que C26.SN.01 à .09 (inédits).

\section{A.1. + A.4.4. Éléments tubulaires tronconiques + éléments annulaires}

Identification : partie basse de montant de lit (pl. 2, $\left.\mathrm{n}^{\circ} 2\right)$

Inventaire provisoire : SN.C26.09

Inventaire Arditi : 672 ?

Provenance : Pompéi

Dimensions : H. max. 94,1; diam. ext. max. 50,1

Détermination : ivoire, fer

Description : élément fragmentaire de montant de meuble, composé d'une âme de fer en partie interne, partiellement creuse, et de quatre pièces ornementales en ivoire. $\mathrm{Au}$ dessus de la partie visible de l'âme de fer (au niveau du bas du fragment) est enfilée une pièce presque complète de forme tronconique, dont la partie inférieure forme un fin tenon surmonté d'une délicate moulure en arête, qui laisse ensuite la paroi remonter en s'élargissant en courbe concave, pour se terminer par un bord supérieur légèrement mouluré et plat sur la tranche. Sur ce tronc de cône repose une bobine simple, s'encastrant ensuite dans un anneau épais, encadré par une deuxième bobine audessus. Le fer de l'âme est tordu à partir de la première bobine, peut-être sous l'effet de la chaleur. Une étiquette incomplète et à moitié effacée indique $6 ? 2$, correspondant à l'inventaire Arditi.

Références comparatives : de nombreux fragments comparables sont en cours d'étude au Musée archéologique de Naples et dans les réserves du site de Pompéi (inédits). 


\section{A.5. Élément sculpté à décor figuré}

Inventaire général Fiorelli : 78295

Inventaire San Giorgio : 7897

Provenance : Pompéi

Dimensions : H. max. 78 ; 1. max 32 ; ép. max. 16

Détermination : ivoire

Description : Élément cylindrique fragmentaire sculpté, représentant un personnage en pied. La partie avant, en bas-relief marqué, a conservé le corps depuis le bas des jambes jusqu'à l'épaule. L'ensemble est recouvert d'un vêtement drapé, dont un pan repose sur le bras gauche, lui-même nu et à demi replié, s'orientant vers la droite. La main, retombant gracieusement et nonchalamment, tient elle aussi une partie du drapé. L'absence de l'extrémité basse et surtout de celle du visage rend incertaine l'identification du personnage, mais il pourrait s'agir d'une danseuse, ou d'une ménade. Le revers du fragment est creux et comporte des traces d'oxydation, indiquant la présence d'un élément métallique, l'âme de fer d'un montant de lit, qui servait d'armature, sur laquelle étaient enfilés les volumes décoratifs.

Références comparatives: d'autres éléments comparables sont en cours d'étude au Musée archéologique de Naples et dans les réserves du site de Pompéi (inédits). Voir par ailleurs en Feugère, Prévot 2008, fig. 7 p. 238 (le fragment, dont le personnage se rapproche à certains égards de celui de Pompéi, est interprété comme un placage de pyxide ou de coffret, mais sans certitude).

\section{B. Le cadre}

\section{B.1. Appliques et garnitures en bandeau}

Identification : applique décorative en bandeau à décor géométrique (pl. $\left.2, \mathrm{n}^{\circ} 4\right)$

Inventaire provisoire : SN.C23.16

Provenance : Pompéi ?

Dimensions : L. max. 49,5; 1. max 23,1; ép. max. 3,1

Détermination : ivoire ou os?

Description: trois fragments assemblés d'applique décorative en bandeau, ornée en bas-relief de motifs géométriques en frise à rais-de-cœur, qui rehaussait le cadre d'un lit. Le revers a conservé les stries de râpe de l'artisan, permettant en particulier une bonne tenue de la colle.

Références comparatives: d'autres éléments comparables sont en cours d'étude au Musée archéologique de Naples et dans les réserves du site de Pompéi (inédits).

27 Identification : garniture en appliques décoratives incrustées ( $\mathrm{pl} .2, \mathrm{n}^{\circ} 5$ )

Inventaire provisoire : SN.C30.06

Provenance : Pompéi

Dimensions : L. max. 87,5; 1. max 24 ; ép. max. 2,4

Détermination : ivoire, pâte de verre, fil d'or

Description: quarante fragments d'appliques décoratives plates (seuls quatre sont présentés en fig. 5), pourvues de fins ornements à motifs végétaux en incisions et en 
incrustations de pâte de verre. Les motifs représentent des feuilles et des fleurs stylisées, et les incisions, en forme de tiges ondulées et de motifs également floraux ou en volutes, étaient comblées de fils d'or, comme le montre l'une d'entre elles qui en est encore pourvue. L'ensemble était manifestement circonscrit par un cadre signifié par une incision linéaire, dont l'un des angles est conservé. Les cernes d'âge de l'ivoire ont par endroit provoqué des cassures incurvées. Le revers porte les traces du ciseau de l'artisan. Environ deux cents incrustations, à l'état fragmentaire pour la plupart, sont également conservées, dont certaines présentent des recouvrements en feuille d'or.

Références comparatives: Les nombreux fragments de cet ensemble n'ont pour le moment trouvé aucune occurrence équivalente.

\section{Le fulcrum}

\section{C.1. Partie basse : le médaillon}

Identification : médaillon décoratif figuré (pl. 2, $\mathrm{n}^{\circ}$ 7)

Inventaire général Fiorelli : 109901

Provenance : Pompéi

Dimensions : H. max. 54 ; 1. max 45 ; ép. max. 9

Détermination : ivoire

Description : élément fragmentaire figuré, sculpté en bas-relief et représentant une tête de femme vue de profil, côté droit. Les lignes du visage sont nettement dessinées, depuis le menton saillant, les lèvres charnues, les naseaux arrondis, le nez droit, jusqu'aux yeux ceints de paupières amples. La chevelure, épaisse sur l'arrière de la tête, semble être retenue en chignon. La partie supérieure de la tête est manquante ainsi que l'arrière, de même que le cou. Il s'agit manifestement d'une décoration de médaillon ornant la partie basse d'un fulcrum de lit.

Références comparatives: d'autres éléments comparables sont en cours d'étude au Musée archéologique de Naples et dans les réserves du site de Pompéi (inédits).

\section{C.3. Partie haute : le couronnement}

Identification : couronnement de fulcrum figuré (pl. 2, $\mathrm{n}^{\circ} 6$ )

Inventaire provisoire : SN.C23.15

Provenance : Pompéi

Dimensions : H. max. $61 ; 1$. max 23,2 ; ép. max. 6,2

Détermination : ivoire

Description : élément fragmentaire d'applique, pourvu d'une décoration en bas-relief représentant le bas d'un oiseau aquatique, avec la patte droite et la queue. Le reste du corps est manquant. La partie conservée comprend sur l'extrémité inférieure la double vague caractéristique constitutive de la liaison avec la partie centrale d'un fulcrum de lit. Le revers plat comporte les traces du ciseau dentelé de l'artisan, dont les lignes creuses permettent une meilleure tenue de la colle. L'observation de la texture et de la couleur de ce fragment laisse entendre qu'il est sans doute passé par les flammes d'un bûcher, et qu'il provient par conséquent probablement d'un contexte funéraire.

Références comparatives: Cormier 2015, vol. II, p. 158. Aucune autre occurrence comparable en ivoire n'est à ce jour connue. 


\section{Conclusion} décorations de lits, une nette et claire frontière entre l'ivoire et l'os. De fait, ces deux matériaux étaient utilisés, nous l'avons dit, pour la fabrication et/ou la décoration de types d'objets équivalents. Mais la comparaison trouve ses limites, car l'ivoire comme matière première coûtait infiniment plus cher que l'os, qu'il était facile d'obtenir, au sortir des marchés aux viandes par exemple, probablement même gratuitement. L'ivoire était en revanche dépendant des importations ${ }^{26}$, et il semble même que la quantité disponible sur le marché ait subi des fluctuations selon les époques; si l'on en croit Pline par exemple, la sienne - et par conséquent les derniers temps de Pompéi vivait une pénurie :

Quamquam nuper ossa etiam in laminas secari coepere paenuria. Etenim rara amplitudo iam dentium praeterquam ex India reperitur ; cetera in nostro orbe cessere luxuriae.

Cependant, faute d'ivoire, on s'est mis récemment à couper en lamelles les os euxmêmes. En effet, il est rare de trouver aujourd'hui de grandes défenses, excepté dans l'Inde; dans notre partie du monde, tout l'ivoire qui s'y trouvait a disparu, absorbé par le luxe ${ }^{27}$.

De plus, la question de l'artisanat et des lieux de production reste mal connue : il a été avancé que l'ivoire était travaillé par les mêmes artisans que ceux qui œuvraient sur $l^{\prime} \mathrm{os}^{28}$; mais il serait hâtif de généraliser. Les textes et inscriptions mentionnent des artisans nommés eborarius ${ }^{29}$ et eboris faber ${ }^{30}$, mais pas ossis faber. Un eborarius pouvait fort bien travailler l'os, mais un travailleur de l'os n'œuvrait pas nécessairement sur l'ivoire, selon le type de clientèle à laquelle son art et sa renommée s'adressaient. Si Pline parle de l'ivoire, c'est parce qu'il s'agit d'un matériau de grande valeur et très prisé, et qu'il est associé aux autres matériaux de luxe sur lesquels il s'attarde justement parce qu'ils sortent de l'ordinaire. L'immense majorité de la population n'était pas concernée par les objets et mobiliers en ivoire, de même qu'aujourd'hui les œuvres d'art qui ont une valeur marchande élevée ne touchent qu'une population réduite, d'amateurs ou de riches collectionneurs ou mécènes. Il a toujours été de bon ton de posséder un tableau d'un artiste en vue, de même qu'à Rome il était remarqué lorsque l'on possédait ou achetait une table en bois précieux incrustée d'ivoire et d'un prix exorbitant, au point que la littérature elle-même pouvait le rapporter ${ }^{31}$. Les proportions ne sont évidemment pas les mêmes à Pompéi qu'à Rome, mais l'idée est la même. Et l'os est un bon moyen d'imiter à moindre coût le luxe de l'ivoire. Le soin pris pour la fabrication des décorations sophistiquées de certains lits en os (pl.1, inv. 112291-112292 ; pl. 2, inv. 78297, 122945, SN.C23.11, 124135), que l'on pouvait par exemple recouvrir de feuilles d'or ${ }^{32}$, peindre ou blanchir de manière à le faire ressembler à l'ivoire, ou dont la qualité technique, voire artistique, était indéniable, montre bien que même avec de tels ornements dorés, le prix ne savait atteindre celui d'un lit du même type fait ou recouvert d'ivoire.

Pour ce qui concerne les lits en ivoire de Pompéi et leurs morphologies, ce paramètre doit être examiné avec nuance, en particulier pour ceux dont les montants sont constitués de volumes lisses, sans décorations figurées ou géométriques (pl.4, inv. SN.C26.09, 78358, 78365). Ne l'oublions pas, Pompéi était une ville moyenne dont l'importance économique et la richesse étaient moindres que celles d'autres cités de la région, comme Cumes ou Naples. La conséquence pour nous, en toute logique, est d'appréhender la notion de luxe avec précaution, en prenant en compte certaines 
variables d'ajustement, en particulier pour l'os ouvragé. À ce titre, l'étude des ivoires et des os du Musée archéologique de Naples se heurte pour le moment essentiellement sur l'obstacle de la contextualisation des pièces et fragments, qui pourraient nous donner de précieuses informations sur leurs propriétaires et sur leur datation. La consultation des archives et journaux de fouilles permettra pour certains d'en affiner la datation et la provenance, ainsi que leur origine sociale. En définitive, cette étude a pour but de rassembler l'ensemble des données disponibles, augmentée par une étude des traces et des techniques du travail artisanal, pour donner une cohérence et proposer une répartition typologique et fonctionnelle, mais également sociale et culturelle, aussi précise que possible.

\section{BIBLIOGRAPHIE}

Allison 2006 = P.M. Allison, The Insula of the Menander at Pompeii. Vol. 3, The finds, a contextual study, Oxford, 2006.

Baldelli 2010 = G. Baldelli, Fanum Fortunae. Recinti funerari e urne lapidee nella necropoli di Via Roma. Notizia preliminare, dans Nuovi Studi Fanesi, 23/24, 2010-2011, p. 7-26.

Bellini 2008 = G.R. Bellini, Un nuovo rinvenimento da Aquinum: il letto in osso della tomba 6, dans M. Sapelli Ragni (dir.), Tra luce e tenebre. Letti funerari in osso da Lazio e Abruzzo, cat. exp. Tivoli, Villa Adriana 24 avril-2 novembre 2008, Milan, 2008, p. 39-48.

Bellini - Montanelli - Trigona 2014 = G.R. Bellini, E. Montanelli, S.L. Trigona, $\mathrm{I}$ letto funerario di Aquinum, dans V. D'Ercole, A. Milaneschi (dir.), I letti funerari in osso dell' Italia centrale, Atti del Seminario di Studi, Roma, Complesso monumentale di San Michele, 3 luglio 2013, Bollettino di archeologia on line, Direzione generale per le antichità V, 3-4, 2014, p. 139-148.

Béraud - Gébara 1986 = I. Béraud, C. Gébara, Les lits funéraires de la nécropole gallo-romaine de SaintLambert (Fréjus), dans RAN, 19, 1986, p. 183-210.

Bertand $2008=\mathrm{I}$. Bertrand (dir.), Le travail de l'os, du bois de cerf et de la corne à l'époque romaine : un artisanat en marge ?, Montagnac, 2008 (Monographies Instrumentum, 34).

Bianchi $2000=$ Ch. Bianchi, Cremona in età romana. I letti funerari in osso dalla necropoli di S. Lorenzo, Milan, 2000.

Bianchi $2008=$ Ch. Bianchi, Letti funerari in osso di età romana : aspetti della produzione e diffusione alla luce di alcuni rinvenimenti in Lombardia. Presentazione preliminare di un letto da Cerveteri (Roma), dans Bertrand 2008, p. 311-334.

Bianchi $2010=$ Ch. Bianchi, $I$ letti con rivestimento in osso e avorio. Analisi dei rinvenimenti dai contest di abitato e funerari, dans LANX, 5, 2010, p. 39-106.

Borriello 1986 = M.R. Borriello, Gli avori e gli ossi lavorati, dans Le Collezioni del Museo Nazionale di Napoli, Naples, 1986, p. 111-112 ; cat. p. 230-233.

Bovero 2014 = A. Bovero et al., Studio e metodologiaper l'intervento di restauro su un letto in osso proveniente dalla tomba $n .5$ di Navelli (AQ), dans V. D’Ercole, A. Milaneschi (dir.), I letti funerari in 
osso dell' Italia centrale, Atti del Seminario di Studi, Roma, Complesso monumentale di San Michele, 3 luglio 2013, Bollettino di archeologia on line, Direzione generale per le antichità V, 3-4, 2014, p. 73-90.

Brun - Munzi 2011 = J.-P. Brun, P. Munzi, Cumes (Italie). Les fouilles du Centre Jean-Bérard 2000-2010, dans RA, 51-1, 2011, p. 147-172.

Caretta $2014=\mathrm{L}$. Caretta, Un letto funerario in osso dalla necropoli meridionale di Falerii Novi (NEPI, VT), dans V. D’Ercole, A. Milaneschi (dir.), I letti funerari in osso dell' Italia centrale, Atti del Seminario di Studi, Roma, Complesso monumentale di San Michele, 3 luglio 2013, Bollettino di archeologia on line, Direzione generale per le antichità V, 3-4, 2014, p. 127-138.

Ciani 1997 = N. Ciani, Tomba 92. 17: Elementi di rivestimento di letto funerario, dans C. Terzani, M.M. Chiari (dir.), Isernia. La Necropoli romana in località quadrella, Rome, 1997, p. 162-169 et 243-245.

Colivicchi 2002 = F. Colivicchi, La necropoli di Ancona (IV-I sec. a.C.). Una comunità italica fra ellenismo e romanizzazione, Loffredo Editore, Naples, 2002, VI - Cat. p. 299-302, 314-324 ; VII - Il sistema rituale (la fase tardo-repubblicana), p. 440-445; p. 462.

Colivicchi 2005 = F. Colivicchi, 28 - Letto con rivestimento d'osso, dans G. de Marinis (dir.), Arte romana nei Musei delle Marche, Ancône, 2005, p. 53.

Colombo - Foresta 2003 = D. Colombo, S. Foresta, L'età romana. L'osso lavorato (tombe 13, 166 et 469), dans E. Laforgia (dir.), Il Museo archeologico di Calatia, Naples, 2003, p. 211-217.

Copersino 2001 = M.R. Copersino, Letti in osso dal territorio di Aielli, dans A. Campanelli (dir.), Il Tesoro del Lago, L'archeologia del Fucino e la Collezione Torlonia, Pescara, 2001, p. 242-246.

Cormier 2015 = A. Cormier, Les lits ornés d'ivoire de Cumes. Art et Mémoire dans les funérailles aristocratiques romaines, $I^{e r}$ s. av. $-I^{e r}$ s. apr. J.-C., thèse de doctorat, université de Paris-Nanterre, 2015.

Cormier 2019 = A. Cormier, Les ivoires et les os ouvragés, dans W. Van Andringa, Th. Creissen, H. Duday (dir.), Naissance et développement d'un paysage funéraire romain ( $I^{e r}$ siècle av. - $I^{e r}$ siècle apr. J.C.). Aux portes de la ville et le long de la route de Nocera, Pompéi. Fouille de la nécropole romaine de Porta Nocera, École française de Rome, université de Lille III, Soprintendenza archeologica di Pompei, Institut Universitaire de France, rapport d'opération 2019, p. 471-528.

Cormier 2020a (à paraître) = A. Cormier, La mémoire des morts : les lits ornés d'ivoire dans le monde romain, Naples, 2020.

Cormier $2020 \mathrm{~b}$ (à paraître) = A. Cormier, Les lits funéraires ornés de stuc et d'os travaillé, dans J.P. Brun, P. Munzi (dir.), Fouilles du Centre Jean Bérard à Cumes, vol. 4 : Les sépultures et les mausolées romains de la zone E, Naples, 2020.

Cormier 2020c (à paraître) = A. Cormier, Les éléments en os d'un lit funéraire, dans M.R. Borriello, J.P. Brun, P. Caputo et al. (dir.), La necropoli del Fondo Artiaco a Cuma. Della «Tomba a tholos» al «Mausoleo delle Teste di cera», Naples, 2020.

Costamagna 2014 = L. Costamagna, I letti dell'alta valle del Nera (PG): rinvenimenti recenti e proposte di rilettura dei vecchi scavi, dans V. D’Ercole, A. Milaneschi (dir.), I letti funerari in osso dell' Italia centrale, Atti del Seminario di Studi, Roma, Complesso monumentale di San Michele, 3 luglio 2013, Bollettino di archeologia on line, Direzione generale per le antichità V, 3-4, 2014, p. 113-126.

De Carolis 2007 = E. De Carolis, Il mobile a Pompei ed Ercolano. Letti, tavoli, sedie e armadi. Contributo Alla tipologia dei mobili della prima età imperiale, Rome, 2007.

De Romanis 2014 = F. De Romanis, Ivory from Muziris, ISAW papers 8, New York, 2014. 
Giovannini 2002 = A. Giovannini, Letti funerari da Aquileia, dans M. Buora, W. Jobst (dir.), Roma sul Danubio: da Aquileia a Carnuntum lungo la via dell'ambra, Rome, 2002, p. 227-229.

Khan 2014 = B. Khan, L'artisanat des matières dures animales : nouvelles connaissances à partir des rebuts d'atelier, dans S. Ferjani (dir.), Matières premières et gestion des ressources, Paris, 2014.

Khan 2019 = B. Khan, L'exploitation artisanale des matières dures d'origine animale au Proche-Orient entre le III ${ }^{e}$ s. av. J.-C. et le VII ${ }^{e}$ s. apr. J.-C. : une approche techno-économique, thèse de doctorat, université Paris 1 Panthéon-Sorbonne, 2019.

Khan - Picod $2018=$ B. Khan, Ch. Picod, Le silence des anneaux : une tentative de reconstitution d'une chaîne opératoire de fabrication d'anneaux en os, dans Artefact, 72018.

Letta 1984 = C. Letta, Due letti funerari in osso dal centro italico romano della Valle d'Amplero (Abruzzo), Rome, 1984, p. 66-115 et pl. I-IX.

Morisco 2012 = M. Morisco, Gli Inventari del Museo Archeologico Nazionale di Napoli, dans Rivista di Studi Pompeiani, 23, 2012, p. 103-108.

Pagano - Prisciandaro 2006 = M. Pagano, R. Prisciandaro, Studio sulle provenienze degli oggetti rinvenuti negli scavi borbonici del Regno di Napoli: una lettura integrata,coordinata e commentata della documentazione, Castellammare di Stabia, 2006.

Pasqui 1907 = A. Pasqui, S. Vittorino. Tomba d'età romana scoperta presso l'antica città di Amiternum, dans NSA, 1907, p. 145-151.

Prévot $2009=\mathrm{Ph}$. Prévot, Caractérisation des sites ruraux de Gaule méridionale : la production et la consommation des objets en os, ivoire et bois de cerf, dans Ph. Leveau et al. (dir.), Les formes de l'habitat rural gallo-romain. Terminologies et typologies à l'épreuve des réalités archéologiques, Bordeaux, 2009 (Aquitania Supplément, 17), p. 47-60.

Prévot 2013 = Ph. Prévot, Éléments de placages et objets en os dans les bûchers, dans W. Van Andringa, H. Duday, S. Lepetz (dir.), Mourir à Pompéi. Archéologie du rituel. Fouilles d'un quartier funéraire de la nécropole romaine de Porta Nocera, Rome, 2013 (Collection de l'EFR, 468), p. 1269-1362.

Riccomini 2013 = A.M. Riccomini, Elementi d'arredo di una stessa officina, dans E. Micheletto, G. Pantò (dir.), Tesoro di Marengo, Museo di Antichità di Torino, Turin, 2013, p. 36-53.

Rodet-Belarbi 2018 = I. Rodet-Belarbi, La transformation des matières dures d'origine animale en Gaule romaine : ateliers urbains et artisans itinérants, dans Artefact, 7, 2018.

Rodziewicz 2007 = E. Rodziewicz, Bone and ivory carvings from Alexandria: French excavations 1992-2004, Le Caire, 2007.

Rossi - Savi 2012 = D. Rossi, F. Savi, I letti in osso lavorato: una prima analisi, dans D. Rossi (dir.), Sulla via Flaminia. Il mausoleo di Marco Nonio Macrino, Milan, 2012, p. 134-161.

Sordini 1893 = G. Sordini, VI. Bolsena. Nuove scoperte nella necropoli di Barano, presso la città, dans NSA, 1893, p. 64-68.

St Clair 2003 = A. St Clair, Carving as craft. Palatine East and the Greco-Roman bone and ivory carving tradition, Baltimore, 2003.

Talamo 1987-1988 = E. Talamo, Un letto funerario da una tomba dell'Esquilino, dans BCAR, 92, 1987-1988, p. 17-102.

Vaglieri 1912 = D. Vaglieri, Ostia. Tomba repubblicana, dans NSA, 9, 1912, p. 95-101.

Zanella 2017 = S. Zanella, Archives de fouilles de Pompéi : mode(s) d'emploi. Un parcours dans les archives, dans S. Zanella, J.-P. Brun et al., Les archives de fouilles : modes d'emploi, Paris, 2017. 
Zanella 2019 = S. Zanella, La caccia fu buona. Pour une histoire des fouilles à Pompéi de Titus à l'Europe, Naples, 2019.

\section{NOTES}

1. Voir par exemple Zanella 2019.

2. Objet d'une thèse de doctorat (Cormier 2015), dont la publication est en cours (Cormier 2020a, à paraître).

3. Brun - Munzi 2011 ; Cormier 2020b (à paraître).

4. Voir par exemple D’Agostino 1985 ; De Filippis Cappai 1997.

5. Cet axe de recherche intègre le matériel conservé dans les dépôts du site de Pompéi, en particulier celui de « Casa Bacco».

6. De Franciscis 1963.

7. Borriello 1986, p. 111-112 ; cat. p. 230-233.

8. De Carolis 2007.

9. Voir à ce sujet la notice éclairante de M. Morisco (Morisco 2012).

10. Allison 2006.

11. Prévot 2013.

12. Cormier 2019. Les méthodes minutieuses employées dans ces fouilles, menées sous la direction de W. Van Andringa, Th. Creissen et H. Duday, permettent une étude très fine des gestes liés aux funérailles, et par conséquent du mobilier attaché aux défunts : non seulement des lits funéraires ornés d'ivoire ou d'os ouvragé, mais aussi des coffrets déposés et des petits ustensiles, que l'on retrouve par ailleurs dans les demeures des vivants.

13. Un nombre équivalent a été étudié dans les dépôts de Pompéi.

14. Cette dernière estimation repose non seulement sur une évaluation visuelle, mais aussi sur la prise en compte et la consultation presqu'achevée des archives et des inventaires.

15. Cormier 2015, p. 115-147, et 2020a : une typologie nouvelle y est proposée, incluant les formes les plus anciennes connues dans le monde méditerranéen, et aboutissant à cette filiation multiforme à l'époque romaine.

16. Cette dénomination connaît un certain flou au départ, lors de la transition progressive d'avec les lits de type hellénistique.

17. Notons cependant le fameux exemplaire en argent du trésor de Marengo (Riccomini 2013, p. 36-53).

18. Voir ici en pl. 1, $\mathrm{n}^{\text {os }} 5,6,7$, et en pl. 2, $\mathrm{n}^{\text {os }} 4,5,6,7$.

19. Plusieurs cas ont été étudiés, qui constituent des bases comparatives solides (sont cités ici quelques exemples notables) : en Gaule surtout (Bertrand 2008, qui rassemble plusieurs études minutieusement documentées, Prévot 2009, Rodet-Belarbi 2018), et quelques-uns en Italie (St Clair 2003), ou d'autres dans le monde grec et au Proche-Orient (Khan 2014; 2019; Khan Picod 2018).

20. Voir par exemple les cas d'Acquasparta en Monacchi 1990-1991, fig. 12, p. 105.

21. Voir par exemple le lit d'Aquinum (Bellini 2008), celui d'Amplero (Letta 1984), ou celui en bronze d'Amiternum conservé à Chieti (Pasqui 1907).

22. Les fouilles du Centre Jean Bérard (USR 3133 CNRS-EFR) dans la nécropole romaine de Cumes ont mis au jour les restes en os de deux lits funéraires, datés du $\mathrm{I}^{\mathrm{er}} \mathrm{s}$. apr. J.-C., qui ne comportent aucune décoration figurée, géométrique ou végétale. Ces lits sont en cours de publication (Cormier 2020b et 2020c). De même le lit orné d'ivoire d'un des grands mausolées de cette même nécropole, qui ne comporte pas non plus de décoration sculptée sur les montants (Cormier 2015, 
vol. II, p. 163). Voir en dernier lieu Sordini 1893 pour le lit de Bolsena, ou bien Colivicchi 2005, p. 53 pour un des lits d'Ancône, dont les montants sont sans décoration.

23. Voir note 13.

24. Au II ${ }^{\mathrm{e}}$ s. apr. J.-C., le commerce de l'ivoire brut est attesté par le papyrus dit « de Muziris » (PVinbod. G. 40822), qui rapporte que le navire Hermapollon transporta entre Coptos et Alexandrie des marchandises importées d'Inde par un port de la Mer Rouge et précise la valeur monétaire des trois quarts de ces biens : parmi ceux-ci, une importante quantité d'ivoire. Fait remarquable, cet ivoire convoyé est composé de deux catégories, que Federico De Romanis, dans un article récent (De Romanis 2014), précise ainsi : d'une part, des $\sigma x i ́ \delta \alpha l$ (« schidai »), morceaux probablement de médiocre qualité délibérément embarqués sous cette forme, ou bien d'un ivoire sain accidentellement brisé - en tout cas de moindre valeur -, pour un poids de $538,5 \mathrm{~kg}$; de

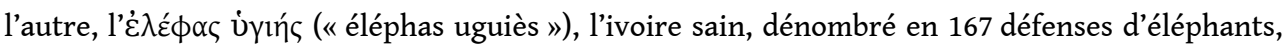
dont le poids indiqué $-3228,5 \mathrm{~kg}-$, représente presque sept fois plus de matière que les «schidai ». La valeur marchande n'est bien sûr pas la même, et permet de supposer par conséquent plusieurs marchés, destinés à la fabrication d'objets de valeurs et de coûts différents.

25. Lorsque aucun numéro d'inventaire ne lui est associé, un numéro provisoire a été attribué à la pièce concernée, incluant d'abord « $\mathrm{SN}$ » pour sans numéro, puis « $\mathrm{Cnn}$ » pour le numéro de la cassette de conservation au musée (C23 par exemple, pour la cassette $n^{\circ} 23$ ), puis un numéro simple d'identification. Les dimensions, qui s'entendent en millimètres, peuvent inclure la longueur maximum (L.), la hauteur (H.), la largeur (l.), la profondeur (prof.), l'épaisseur (ép.), le diamètre interne (diam. int.) et le diamètre externe (diam. ext.).

26. Cormier 2015, vol. I, p. 238-239.

27. H. N., 8, 4, trad. A. Ernout, CUF, 1952.

28. Bonacasa Carra 2000, par exemple, suggère que les objets manufacturés en ivoire et en os proviennent manifestement des mêmes ateliers, avec les mêmes caractéristiques techniques d'exécution, et adoptant le même répertoire décoratif et typologique (p. 253-254).

29. CIL, VI, 7655, 7885, 9375, 9397, 33423, 37374a, 37793 ; IX, 5140. Ces inscriptions sur marbre, découvertes au XIX $\mathrm{X}^{\mathrm{e}}$ siècle, sont difficiles à dater précisément.

30. Horace, Ep., 2, 196 : Marmoris aut eboris fabros aut aeris amauit.

31. Pline (H.N., 13, 29) rapporte qu'une table de citre (c'est-à-dire en thuya) appartenant à Cicéron valait un million de sesterces.

32. Bellini 2008 en particulier, pour le lit d'Aquinum, au sud de Rome en direction de Naples.

\section{RÉSUMÉS}

Cet article fait état de l'avancement d'une partie de l'étude entamée sur les ivoires et les os ouvragés conservés dans les réserves du Musée archéologique de Naples, avec la présentation d'un échantillon d'éléments, intégralement conservés ou fragmentaires, appartenant à des lits issus de contextes domestiques, essentiellement à Pompéi. Seul un décor en ivoire présente les caractéristiques d'un passage par les flammes, témoignage d'un probable bûcher funéraire. Ce choix de pièces représentatives ponctue un travail plus vaste incluant une étude morphologique, typologique et fonctionnelle, puis la création d'un catalogue raisonné sur l'ensemble des ivoires et des os du musée. Mise en regard avec le mobilier du même type conservé dans les réserves du site de Pompéi, l'étude a pour objectif d'examiner cet aspect important de la culture matérielle et 
de proposer un état de la question, du point de vue des usages mais aussi de celui des techniques artisanales, pour une période située entre la fin de la République romaine et la fin $\mathrm{du} \mathrm{I}^{\mathrm{er}}$ siècle apr. J.-C.

This paper put forward the progress of a research on finely-worked ivory and bone preserved in the storerooms of the Archeological Museum of Naples. It presents a sample of entire or fragmentary pieces of beds from domestic contexts, mainly in Pompeii. Among them, only one decor has marks of fire, suggesting that it was burned in a funerary pyre. This selection of representative pieces is part of a lager research including a morphological, typological and functional study, and the creation of a reasoned catalogue of all ivories and bones of the Museum. Comparing them with the same material from the storerooms of Pompeii, the study aims to examine this important aspect of Roman material culture and to offer a state of the art, dealing with the uses as well as craftsmanship, for a period between the end of the Roman Republic and the end of the first century AD.

INDEX

Mots-clés : ivoire, os ouvragé, artisanat, tabletterie, lit domestique, lit funéraire, Pompéi Index géographique : Pompéi, Naples institutions FNRS/CReA-Patrimoine (Centre de Recherches en Archéologie et Patrimoine), Université libre de Bruxelles, Centre Jean Bérard, Ministère de l'Europe et des Affaires étrangères (Paris), Musée archéologique de Naples

Keywords : ivory, bone carving, craftsmanship, craft, domestic bed, funerary bed, Pompeii

\section{AUTEUR}

\section{ANSELME CORMIER}

FNRS/CReA-Patrimoine (Centre de Recherches en Archéologie et Patrimoine), Université libre de Bruxelles - anselme.cormier@orange.fr 